\title{
An innovation involving self-surveillance and serious gaming to increase smoking quit rate: Protocol for a pilot randomized controlled trial
}

Ngiap C. Tan 1,2, Yi L. E. Koh', Chin C. Goh', Soh H. A. Ngoh' ${ }^{1}$ Ai M. Tan', Usha Sankari', Seng B. Lee³, Hong C. D. Tay', Soon H. Lim ${ }^{4}$

\begin{abstract}
Smoking is a health hazard. Current smoking cessation measures such as behavioral change counselling by trained professionals, nicotine replacement therapy and medications have limited success. Smoking intensity is assessed using a portable device to measure the smokers' exhaled breath carbon monoxide (eCO) level. A systematic review suggests the potential of serious gaming to increase smoking quit rate. However, the related studies were unable to explain and determine the effect gamification on smoking cessation.

A handy personalized eCO measurement device linked to a smart-phone applications (app) has been developed (integrated STEADES-2 system). This novel system incorporates app-based video and print learning resources, authentication function and gamification using the eCO data as game element. Trained multidisciplinary healthcare professionals access the STEADES-2 data to monitor smoking status and support smokers via asynchronous virtual coaching. The pilot randomized controlled trial will enroll 20 smokers to use the STEADES-2 system (intervention group) and another 20 to the existing smoking cessation programme (control group) in primary care. The primary feasibility outcomes will include the recruitment response rate, the smokers' usability of the STEADES-2 system, their self eCO monitoring, frequencies of participation in the serious games and interactions with their virtual coaches. Their smoking literacy, utility and experience of the STEADES-2 system are other outcomes. Smokers in both groups will be compared on their cigarette abstinence as secondary outcome based on eCO levels and urine cotinine test (primary outcomes after 12 weeks). The results will be disseminated via conferences and publications.
\end{abstract}

\section{AFFILIATION \\ 1 SingHealth Polyclinics, Singapore 2 SingHealth-Duke NUS, Family Medicine Academic Clinical Program, Singapore 3 Taggle Private Ltd, Singapore 4 Institute of Technical Education College West, Singapore}

\section{CORRESPONDENCE TO}

Ngiap C. Tan. SingHealth

Polyclinics, 167 Jalan Bukit Merah, Connection One, Tower 5, \#15-10, 150167, Singapore. Email: tan. ngiap.chuan@singhealth.com.sg ORCID ID: https://orcid.org/00000002-5946-1149

\section{KEYWORDS}

smoking cessation, carbon monoxide, coach, surveillance, serious games, innovation

Received: 10 December 2020 Revised: 10 May 2021

Accepted: 14 June 2021

\section{INTRODUCTION}

Smoking is a global public health problem. Measures to curb smoking are multi-pronged. At the personal level, supporting smokers' behavior change by trained healthcare professionals has shown positive results but requires intensive resources for their continuous engagement ${ }^{1-4}$. Therapeutic agents such as nicotine replacement to reduce withdrawal symptoms and psychotropic medications facilitate smoking cessation ${ }^{5-7}$, but only if smokers are ready to quit smoking. At the system level, the outcomes of smoking cessation programmes incorporating these interventions are variable due to multiple barriers ${ }^{8,9}$. Dahne et al. ${ }^{10}$ have reported the need to keep up with new technologies in tobacco-focused clinical trials that some people prefer to use such as personal 
carbon monoxide monitors, smoking cessation apps or programs ${ }^{10}$.

One major difficulty is the lack of reliable and convenient monitoring by healthcare professionals to track the cessation efforts of the smokers. Selfreporting may not be entirely reliable. The use of a device to measure exhaled breath carbon-monoxide (eCO) level enables monitoring of smoking cessation progress ${ }^{11}$. The level of eCO in ppm corresponds to the percentage of carboxyhemoglobin or the percentage of blood cells carrying $\mathrm{CO}$ instead of oxygen. It is simple to execute and has been shown to be a reliable, valid and acceptable device to distinguish smokers from non-smokers ${ }^{10}$. The clinical use of $\mathrm{CO}$ testing has been shown to provide biomedical feedback on smoking behavior, educate smokers on tobacco health hazards, aid treatment planning, and serve as a motivational tool to encourage people to become tobacco free ${ }^{12,13}$. Beard and $\mathrm{West}^{14}$ had reported that frequent and personal use of an eCO meter resulted in more effective smoking cessation in their pilot trial. The iCO TM Smokerlyzer ${ }^{\circledR}$ manufactured by Bedfont ${ }^{\circledR}$ Scientific Ltd is an example of an eCO meter which is available for purchase. Alternatively, the cotinine test is sensitive to determine the nicotine level in saliva or urine of up to 4 days but is expensive ${ }^{15}$.

Both the eCO meter and cotinine test are often available only in healthcare settings. These measures fail to reach out to the smokers at their contemplation stage, when they have yet to seek assistance in clinical settings. For this group of smokers, behavioral and motivational support from trained smoking cessation coaches may shift their ambivalence towards affirmative decision to quit. In this Internet of Things era, such support and nudges can be delivered remotely and asynchronously from virtual coaches via mobile technology.

Smokers' views of a personal eCO meter have been favorable with high expectations of their potential to increase motivation. Herbec et al. ${ }^{16}$ had reported that smokers considered easy eCO testing, relevant and motivating feedback, and recording of contextual data as priority features of such a device and associated app system. Appearance and usability of the personal CO monitor, and accuracy and relevance of $\mathrm{CO}$ testing were considered important for engagement.
Another recent development is the application of 'serious games' in healthcare. Marsh ${ }^{17}$ defines 'serious games' as digital games, simulations, virtual environments and mixed reality/media that provide opportunities to engage in activities through responsive narrative/story, gameplay or encounters to inform, influence, for well-being and/ or experience to convey meaning. Serious games have been applied in disease prevention, diagnosis and treatment of a great variety of health behaviors and disease ${ }^{18-21}$. A randomized controlled trial of $\mathrm{Nicot}^{22}$, a video game in which players crush virtual cigarettes in a 3D game environment, reported $13 \%$ increase in smoking cessation rates compared with a balloon popping game. Derksen et al..$^{23}$ have shown favorable outcomes in smoking cessation associated with serious gaming in their recent systematic review. However, they reported that most studies in the systematic review had important methodological limitations, which failed to adequately demonstrate, quantify, and understand the effects of serious games on the outcomes ${ }^{23}$.

Portable CO meters or monitoring devices such as $\mathrm{iCO} \AA$, have been used in earlier trials to assess smoking quit rate. Herbec et al. ${ }^{16}$ had demonstrated unsuccessful use of CO meters to remotely assess abstinence using a smartphone stop-smoking app in their pragmatic trial. One possible reason for the failure could be related to the requirement of the eCO meter to be linked to a personal computer after installation of software. Krishnan et al. ${ }^{24}$ have also shown no significant differences in smoking cessation, smoking reduction, and motivation to quit between study arms over a 30-day period in their randomized controlled trial of a mobile phone-based messaging support (Coach2Quit) and eCO measurement using the Bedfont ${ }^{\circledR}$ iCO meter. While $91 \%$ of the smokers liked the Coach2Quit app, messaging support alone may be inadequate compared to individual counselling by trained professionals to address specific personal and contextual issues faced by the smokers.

Earlier failures in innovation-based interventions are important lessons. The need for design-thinking of novel system to facilitate smoking cessation is pertinent. The Pivot smoking cessation program has been developed, leveraging on a personal portable eCO meter, smartphone app and in-app text-based 
coaching. It shows that about one-third of the smokers quit smoking at the end of the program ${ }^{25}$. We had developed and proven the acceptability of Asian smokers using a portable eCO measurement device, called STEADES-1, to self-monitor their smoking cessation efforts, and to transmit their eCO data to their family members and friends in an earlier feasibility study ${ }^{26}$.

We have now created an enhanced prototype, STEADES-2, with features and functionalities to bridge known gaps in literature. Similar to the iCO TM Smokerlyzer ${ }^{\circledR}$, it includes additional authentication functionality to reduce fraudulent use of the device and is linked by Bluetooth to the STEADES app without a need to connect with a computer. The app embeds a repository of educational resource material and a purposefully designed STEADES game. The serious game uses a contingency management approach which leverages on game-based rewards to incentivize smoking abstinence instead of the monetary rewards ${ }^{27}$. The app also allows remote asynchronous interaction with virtual coaches in addition to automated push-through motivational text messaging. The eCO meter, mobile app, serious games and virtual coaching are integrated in the STEADES-2 system.

The mixed outcomes in innovation-based interventions necessitate a feasibility evaluation of the STEADES system in a pragmatic trial. A smallscale trial, such as those conducted by Herbec et al. ${ }^{16}$, is less costly due to shorter completion time from smaller number of subjects. Nevertheless, it will provide insight into the uptake and usability of the STEADES-2 system effect size of the complex intervention and identify any gaps for its further improvement.

\section{Aims}

This pilot randomized controlled trial primarily aims to assess the utility and usability of the STEADES system among the smokers in the intervention arm. Smokers in the control arm are recruited into the existing smoking cessation program at the study site, in which they will undertake face-to-face counselling with a trained nurse. The study also aims to determine the proportion of smokers with total smoking abstinence in the STEADES-2 intervention arm compared to those in the control arm at the end of 12 weeks post-enrolment as secondary outcome.

\section{METHODS \\ Study setting and sites}

The study will be conducted in an ambulatory setting at public primary care clinics (polyclinics) in a densely populated, urbanized community in Singapore.

The main site for the implementation of the study will be located at two branches of SingHealth Polyclinics in Pasir Ris and Bedok estates in northeastern and eastern region of the island state.

\section{Subjects and inclusion criteria}

The target subjects are adult smokers, aged $\geq 21$ years, who will be recruited at the two polyclinics. Smokers from the other SingHealth Polyclinics will be directed to the study sites by internal referral.

The smokers will be screened for the following eligibility prior to their randomization in the trial:

1. Subjects who smoke at least one cigarette per day;

2. Current user of a smartphone to download the STEADES mobile application;

3. Willingness to monitor the eCO at least once daily for smoking cessation using the STEADES-2 device;

4. Willingness to engage the virtual coach at least once weekly during the study period;

5. Willingness to play the serious games at least once daily; and

6. Ability to provide informed consent and to return the STEADES-2 device to the study team upon completion of the study (for intervention group).

\section{Exclusion criteria}

The exclusion criteria are:

1. Non-smoker or ex-smoker who has not smoked any cigarette for the past one month;

2. Current user of mobile phone which lacks the function to download mobile application;

3. Inability to commit to the study completion or return of the STEADES-2 device at the end of the study (for subjects randomized to the intervention group); and

4. Any disability which renders the smoker incapable of providing informed consent independently.

\section{Sample size calculation}

This is planned as a feasibility study to explore the 
recruitment and deployment of a multi-component innovation on smoking cessation. Due to paucity of local data, the effectiveness of the existing smoking cessation programme on quit rate will be evaluated in the control arm in this pilot RCT. This will allow an estimation of an effect size of using the STEADES-2 system, if any, in comparison with the current smoking cessation programme. Julious ${ }^{28}$ has suggested a sample size of 12 per group rule for a pilot study. To address potential dropout, a total of 40 smokers will be recruited in this pilot trial, of which 20 will be randomly allocated to the intervention arm and 20 to the control arm.

\section{Randomization}

Randomization will be carried out centrally at the Research Department office at the institution headquarters. The clinical research coordinator (CRC) will call in to an administrator to determine the assignment of the consented subject. The administrator will use a sealed envelope with a prior sequence of allotment based on random numbers generated by SPSS software to assign the subject to either the intervention or controlled arm.

\section{Recruitment process}

A CRG will be trained by the investigators on the functions and use of the STEADES-2 system. The CRC will recruit smokers who are referred by the clinical teams at the study sites. They will provide information to the potential subjects using the institution review board's approved study documents, which describe the study intent and protocol. The CRCs will screen the potential subjects for eligibility and address their queries before the subjects endorse the written consent form based on Good Clinical Practice guidelines.

\section{Intervention and control arms}

Subjects in the intervention arm will fill in the questionnaire embedded in the STEADES app and will be introduced to the use of the STEADES-2 meter by the CRC. The STEADES meter is developed by the technical team comprising investigators from ITE College West. STEADES-2 measures the amount of eCO in tidal expired breath as a marker for smoking status ${ }^{13}$. People who smoked cigarettes recently have higher levels of CO in their expired breath.
Subjects will be informed that smoking is a serious health hazard. It helps them to realize that harmful CO gathers in their body and the harm dissipates by smoking cessation. Such recognition could potentially motivate them and improve adherence to their smoking cessation efforts. The intent will be to raise awareness about this health risk not only to the smokers themselves, but also to their family members, colleagues and friends when smoking in their presence.

The CRC will assist to download the specially designed STEADES-2 app on the subject's smartphone. Next, the STEADES-2 device is paired to the app in the smart-phone via Bluetooth using facial recognition or digitalized thumb print of the smoker. Whenever the smoker uses the STEADES-2 device, the eCO data are recorded by the app, which is activated by its facial recognition or digitalized thumb-print functionality of the smartphone. In this way the eCO data are authenticated and linked to the smartphone user/owner. The CRC will proceed to demonstrate the various functionalities of the app and the procedure for the serious games; and linkage to the virtual coach. The eCO data from the STEADES-2 meter are transmitted via Bluetooth to the STEADES app. All activities, eCO data and gameplay on the STEADES-2 app will be uploaded to a centralized server (AWS Cloud), where it will be displayed for the virtual coaches to monitor. Through their dashboard in the app, the virtual coaches will be able to communicate and provide advice to the smokers.

The smokers in the intervention arm will be able to:

1. Visualize the eCO trend, activity and smoking cessation progress on a dashboard.

2. Access an inventory of interactive (written and video) learning materials which they can select, based on their personal values, preferences and motivation to quit smoking. The videos last between 32 seconds and 3.5 minutes. The reading material covers various techniques to manage the different obstacles commonly faced by the smokers. The completion of each lesson is tracked by the app for the awareness of the virtual coaches. Quizzes can be administered to test the understanding of the smokers and gather their feedbacks on what they perceive of the STEADES system. 
3. Join the STEADES Club to interact with their virtual coaches and other players. The STEADES Club is modelled after a real-world club, where members gather to meet friends and take part in activities. The concept is adopted from a separate gamification app developed by a study team member. The Club is where the smoker can get information and updates on the programme and talk to his virtual coach for advice and feedback on his smoke cessation performance. The smokers will be prompted to fulfil their daily STEADES related tasks in order to advance in their games. The 20 smokers will be grouped in 5 teams of 4 players in the club to allow intra- and inter-team competition to achieve the game goal.

4. Play the STEADES game by themselves and with other anonymized smokers in the intervention arm. The serious game leverages on major gamification strategies recommended by Cugleman et al. ${ }^{29}$ such as goal setting, capacity to overcome challenges, providing feedback on performance, reinforcement, comparing progress, social connectivity, and fun and playfulness. It embeds Cognitive Behaviour Therapy and concept of selfdetermination to achieve smoking cessation as the goal $^{22}$. Table 1 describes the game content and gamification strategies. The design and content of the STEADES game is to nudge the smokers over 5 stages to reach the castle as the goal, as depicted in Table 2.

5 . Interact asynchronously with their virtual coaches, comprising multidisciplinary primary care professionals including a family physician, three nurses and a pharmacist. They have the training and experience in smoking cessation and will serve as pro bono virtual coaches to provide advice and support to the smokers during the study period. They will be anonymized and generic names like 'Coach Alex and Anna' will be used. They will support the smokers via app-based asynchronous counselling. The coach dashboard in the STEADES app allows the entire team of virtual coaches to view the status of all the participating smokers. This realistic approach enables the coaches to cross-cover each other in view of their work arrangement. They will discuss and coordinate among themselves to personalize the care plan for each smoker via a separate chat group.

The smokers are instructed to complete their daily goals comprising three tasks, with no restriction to the frequencies: 1) measurement of their eCO using the STEADES-2 meter; 2) fill their cigarette log using the app; and 3) check in to their game challenge. They will be rewarded with an 'InvisibleShield' for the game play after completing five logs of 'how smoking will improve their life' and five logs to show 'how badly smoking has affected their life'. This is a deliberate measure for the smoker to reflect on their push and pull motivations to quit smoking. They will be rewarded with a 'Mind-Aura Crown' after engaging their coach, complete remote smoking counselling and attain their game goal. Every use of

\section{Table 1. Gamification strategies in STEADES serious game}

\begin{tabular}{|c|c|}
\hline Gamification strategies & Descriptions \\
\hline Goal setting & $\begin{array}{l}\text { Smokers set date to end their smoking as their main goal. Reaching the castle in the game shares the same } \\
\text { intention. }\end{array}$ \\
\hline $\begin{array}{l}\text { Capacity to overcome } \\
\text { challenges }\end{array}$ & $\begin{array}{l}\text { Smokers have to overcome day-to-day challenges in their quit journey. Similarly, they have to overcome the } \\
\text { Smoke-Demons in the game. }\end{array}$ \\
\hline Feedbacks & $\begin{array}{l}\text { Smokers are asked to monitor their eCO using the STEADES meter and set quit date. They are cognizant of } \\
\text { their victories and failures during the pursuit of their game goal. }\end{array}$ \\
\hline Reinforcement & $\begin{array}{l}\text { The eCO data from the STEADES meter provide additional 'ammunition/bomb' to aid the smokers to overcome } \\
\text { the Smoke-Demons. Game awards such the Invisible-Shield help to shield them from the Smoke-Demons, so } \\
\text { that they can progress with their journey. }\end{array}$ \\
\hline Comparing progress & $\begin{array}{l}\text { The smokers in the club can view the scores of their peers in the teams and the competition aims to nudge } \\
\text { their behavioral change. }\end{array}$ \\
\hline Social connectivity & The smokers can encourage other team players in the club using motivational messages. \\
\hline Fun and playfulness & $\begin{array}{l}\text { Smokers are required to destroy Smoke-Demons as they continue their game journey, which contributes to } \\
\text { their rewards and accelerates their pace of reaching the goal. }\end{array}$ \\
\hline
\end{tabular}




\section{Table 2. The stages of the STEADES game}

Stages Descriptions of equivalent approach in the STEADES game
Seek The player acquires the 'Book of Knowledge' from the repository of learning material and will be awarded the Invisible-Shield
and the Mind-Aura Crown.
Quest The player opens up the 'Golden Path' linking the army to the castle, and to unlock next phase of the journey.
Army The player recruits soldiers to form an army and prepare to combat the Smoke-Demons.
Attack Each day in a week, the player fights against the Smoke-Demons to reach the castle.
Secure Once there is evidence of smoking cessation over 7 days (indicated in the eCO parameter), the player will be able to build
barriers and form new armies to protect the castle.

the STEADES-2 meter and app will be recorded by the system and accessible to the coaches.

In the control arm, the subjects will be enrolled into the existing smoking cessation program in the respective polyclinic. A trained nurse counsellor will provide smoking cessation advice to the smokers and will also assess their exhaled breath analyzed using both a commercially available eCO measurement device and the STEADES-2 device. They will complete a questionnaire and their smoking status will be re-assessed at 12 weeks after their enrolment.

Smokers in both arms will be tested to assess their smoking status by using the urine cotinine test and eCO measurements via the STEADES- 2 device at the point of study enrolment.

\section{Questionnaire}

The questionnaire will collect the following data from the smokers:

1. Demographic characteristics: year of birth, gender, ethnic group, marital status, educational level, employment status, estimated annual personal income.

2. Smoking characteristics: readiness to quit smoking (pre-contemplation, contemplation, preparation, action, maintenance), modified Fagerström test for nicotine dependence ${ }^{30}$; CAGE questionnaire modified for smoking behaviour ${ }^{31}$; 4 Cs test to assess nicotine addiction (compulsion, control, cutting down, withdrawal symptoms, and consequences) ${ }^{32,33}$; smoker's profile (stress relief, conditional responses, relief of withdrawal symptoms, elevation of depression mood); and number of cigarettes smoked per day).

3. Intervention arm only: views and experience with the gamification and the STEADES-2 system;
Games Experience Questionnaire (GEQ) ${ }^{34}$ and System Usability Scale (SUS) ${ }^{35}$.

4. Results of the STEADES-2 measurement and urine cotinine test conducted by the clinical research coordinator.

5. Hypothetical set of questions to gather feedback on the perceived pricing of using a system to support smoking cessation.

The questionnaires will be administered in-person on enrolment and end of the trial at 12 th week. A CRC will assist to fill in the questionnaire (Table 3) over a phone interview at 4 th week post-enrolment.

\section{Completion visit}

The subjects of both arms will make a final study visit at the 12 th week post-enrolment. Their smoking status of the subjects will be determined by on-site measurement of the eCO using their STEADES-2 device as well as to detect the presence of nicotine related product using the urine cotinine test kit. Photo of the STEADES-2 measurement and the cotinine test kit result, together with the study identification number of the subject (excluding identifiable personal data such as facial photo) will be recorded by the CRC to define the outcomes and stored in a secured hard disk for reference.

\section{Primary outcomes}

The feasibility outcomes include the respective scores by the smokers at enrolment and at 12 th week postenrolment:

1. Number of cigarettes smoked per day

2. Change in the smokers' readiness to quit smoking

3. Score of the modified Fagerström test for nicotine dependence $^{30}$

4. Score in the CAGE questionnaire modified for 
Table 3. Characteristics and data collection of participants at three time points

\begin{tabular}{|c|c|c|c|}
\hline Characteristics & Enrolment & 4 weeks & 12 weeks \\
\hline $\begin{array}{l}\text { Demographic characteristics: } \\
\text { year of birth, gender, ethnic group, marital status, highest educational level attained }\end{array}$ & $x$ & & \\
\hline Demographic characteristics: employment status, estimated annual personal income & $x$ & & $x$ \\
\hline $\begin{array}{l}\text { Smoking characteristics: readiness to quit smoking (pre-contemplation, contemplation, } \\
\text { preparation, action, maintenance); modified Fagerström test for nicotine dependence; } \\
\text { CAGE questionnaire modified for smoking behavior; Number of cigarettes smoked per day }\end{array}$ & $x$ & $\mathrm{Xx}$ & $x$ \\
\hline $\begin{array}{l}\text { Smoking characteristics: } 4 \text { Cs test to assess nicotine addiction (compulsion, control, } \\
\text { cutting down, withdrawal symptoms, and consequences); smoker's profile (stress relief, } \\
\text { conditional responses, relief of withdrawal symptoms, elevation of depression mood) }\end{array}$ & $x$ & & $x$ \\
\hline Games Experience Questionnaire (GEO) and System Usability Scale (SUS) & & $\#$ & \# \\
\hline STEADES-2 measurement and urine cotinine test & $\mathrm{x}$ & & $x$ \\
\hline $\begin{array}{l}\text { Hypothetical set of questions to gather feedback on the perceived pricing of using a } \\
\text { system to support smoking cessation }\end{array}$ & $X$ & & $x$ \\
\hline
\end{tabular}

X: apply to both intervention and control arms. Xx: apply to both arms but the data will be collected from phone interview. \#: apply only to intervention arm and embed in the STEADES app or collected by phone interview.

smoking behaviour ${ }^{31}$

5. Smoker's profile

6. Four Cs test ${ }^{32,33}$

7. Scores in the Games Experience Questionnaire $(\mathrm{GEQ})^{34}$

8. Scores in the System Usability Scale (SUS) ${ }^{35}$

9. Perceived pricing of using the system

The scores for 7,8 and 9 will be computed from smokers from the intervention arm only.

\section{Secondary outcome}

Total smoking abstinence is defined as both measurements, using the STEADES-2 device, with results of 0 or $1 \mathrm{ppm}$ and negative cotinine test.

Partial smoking cessation is defined as either a measurement by the STEADES-2 device exceeding $1 \mathrm{ppm}$ or positive cotinine test. The difference in the number of cigarettes smoked on enrolment and at the end of 12 weeks is another indicator of partial cessation.

Failed smoking cessation is defined if STEADES-2 measurement exceeds 1 and positive cotinine test.

\section{Statistical analysis plan}

Descriptive statistics will be presented in frequency and percentages. Independent t-test and MannWhitney U test will be used for normally distributed data and non-normally distributed data, respectively, to compare the difference in scores between the two groups for the following variables: number of cigarettes smoked per day; score of the modified Fagerström test for nicotine dependence ${ }^{30}$; score in the CAGE questionnaire ${ }^{31}$ modified for smoking behavior; 4 Cs scores ${ }^{32,33}$; Scores in the Games Experience Questionnaire (GEQ) $)^{34}$; Scores in the System Usability Scale (SUS) ${ }^{35}$; and options in the perceived pricing of using the system.

Smoking cessation is defined as both measurements using the STEADES-2 device with results of 0 or $1 \mathrm{ppm}$ and negative cotinine test. Smoking cessation will be assessed with demographics using chi-squared test to identify the profiles of those who quit smoking. Smoking cessation between the intervention and control groups will also be compared using chi-squared test. Considering the small study population, cells with $<5$ counts will be compared using Fisher's exact test. All analyses will be done using IBM SPSS version 25.0. A $p<0.05$ is considered significant.

\section{Ethical approval}

The SingHealth Institution Review Board approved the study (CIRB\#: 2020/2762).

\section{Clinical trials registration}

The trial is registered with the clinical trials registry in the United States National Library of Medicine: NCT04630340. 


\section{Implementation time frame}

The STEADES-2 system has been developed and has undergone several rounds of user acceptability testing by the study team members. The trial will be launched in May 2021.

\section{DISCUSSION}

The current approach in smoking cessation is suboptimal in clinical practices due to various challenges. The proposed system focuses on the Internet of Things (IoT) approach in harnessing virtual coaching to motivate smokers to quit their habit. This innovation aims to reach out to a wider pool of smokers, who may not avail themselves to face-to-face consultation in a healthcare setting, which is unpopular, costly and labor intensive. The selfsurveillance of the eCO will allow smokers to reflect on the hazards of smoking and to chart their own progress in smoking cessation. The accessibility to virtual coaching provides the smokers with personcentered advice and counselling, remotely. The IoT can potentially transcend geographical boundaries to reach out to smokers beyond the Singapore border. There is significant commercial potential for enterprise to tap on this innovation to reach out to a global pool of smokers, as access to smartphone is now ubiquitous across the world. It may also prove to be a game changer for insurance companies to design creative incentive-based products for the benefits of their clients who are successful in smoking cessation.

\section{Limitations}

The pilot trial design is limited by the small number of subjects in both arms, which in turn will limit the generalizability of the results. It primarily aims to determine the feasibility of the modus operandi of the entire STEADES-2 system and to gather feedbacks from the smokers in the intervention arm.

\section{CONCLUSIONS}

This pilot randomized controlled trial will provide insight into the feasibility of a triad of self-surveillance of exhaled carbon monoxide measurements, virtual coaching and serious gamification on smoking cessation. The results will determine the association of the profile of smokers, their smoking behavior, utility of the serious games and self-surveillance and interaction with the virtual coach on their smoking quit rate.

\section{REFERENCES}

1. Jepson RG, Harris FM, Platt S, Tannahill C. The effectiveness of interventions to change six health behaviours: a review of reviews. BMC Public Health. 2010;10(1):538. doi:10.1186/1471-2458-10-538

2. Movsisyan NK, Varduhi P, Arusyak H, et al. Smoking behavior, attitudes, and cessation counseling among healthcare professionals in Armenia. BMC Public Health. 2012;12(1):1028. doi:10.1186/1471-2458-12-1028

3. Abdulateef DS, Ali AJ, Abdulateef, DS Mohesh MI. Smoking Knowledge, Attitude, and Practices Among Health Care Professionals from Sulaymaniyah City/Iraq. Tob Use Insights. 2016;9:1-6. doi:10.4137/TUI.S38171

4. Beck RS, Daughtridge R, Slone PD. Physician-patient communication in the primary care office: a systematic review. J Am Board Fam Pract. 2002;15(1):25-38. Accessed May 11, 2021. https://www.jabfm.org/content/ jabfp/15/1/25.full.pdf

5. Xi Z, Spiller K, Gardner E. Mechanism-based medication development for the treatment of nicotine dependence. Acta Pharmacol Sin. 2009;30(6):723-739. doi:10.1038/ aps.2009.46

6. Buchhalter AR, Fant RV, Henningfield JE. Novel pharmacological approaches for treating tobacco dependence and withdrawal: current status. Drugs. 2008;68(8):1067-1088. doi:10.2165/00003495200868080-00005

7. Kenny PJ, Markou A . Neurobiology of the nicotine withdrawal syndrome. Pharmacol Biochem Behav. 2001;70(4):531-549. doi:10.1016/s00913057(01)00651-7

8. Daoud N, Jung YE, Sheikh Muhammad A, et al. Facilitators and barriers to smoking cessation among minority men using the behavioral-ecological model and Behavior Change Wheel: A concept mapping study. PLoS One. 2018;13(10):e0204657. doi:10.1371/journal. pone.0204657

9. Moffatt J, Whip R, Moffatt J. The struggle to quit: barriers and incentives to smoking cessation. Health Educ J. 2004;63:101-112. doi:10.1177/001789690406300202

10. Dahne J, Tomko RL, McClure EA, Obeid JS, Carpenter MJ. Remote Methods for Conducting Tobacco-Focused Clinical Trials. Nicotine Tob Res. 2020;22(12):21342140. doi:10.1093/ntr/ntaa105

11. Meredith SE, Robinson A, Erb P, et al. A Mobile-PhoneBased Breath Carbon Monoxide Meter to Detect Cigarette Smoking. Nicotine Tob Res. 2014;16(6):766-773. doi:10.1093/ntr/ntt275

12. Shahab L, West R, McNeill A. A randomized, controlled trial of adding expired carbon monoxide feedback to brief stop smoking advice: evaluation of cognitive and behavioral effects. Health Psychol. 2011;30(1):49-57. doi:10.1037/a0021821

13. Goldstein AO, Gans SP, Ripley-Moffitt C, Kotsen C, Bars M. Use of Expired Air Carbon Monoxide Testing 
in Clinical Tobacco Treatment Settings. CHEST. 2018;153(2):554-562. doi:10.1016/j.chest.2017.11.002

14. Beard E, West R. Pilot study of the use of personal carbon monoxide monitoring to achieve radical smoking reduction. J Smoke Cessat. 2012;7(1):12-17. doi:10.1017/jsc.2012.1

15. Akiko M, Akane M, Masayoshi I, Payton NM, Hirotaka O, Megumi H. Simultaneous measurement of urinary total nicotine and cotinine as biomarkers of active and passive smoking among Japanese individuals. Environ Health Prev Med. 2013;18(3):244-50. doi:10.1007/s12199-0120307-5

16. Herbec A, Perski O, Shahab L, West R. Smokers' Views on Personal Carbon Monoxide Monitors, Associated Apps, and Their Use: An Interview and Think-Aloud Study. Int J Environ Res Public Health. 2018;15(2):288. doi:10.3390/ijerph15020288

17. Marsh T. Serious games continuum: Between games for purpose and experiential environments for purpose. Entertainment Computing. 2011;2(2):61-68. doi:10.1016/j.entcom.2010.12.004

18. Kato PM. Video games in health care: closing the gap. Rev Gen Psychol. 2010;14(2):113-121. doi:10.1037/ a0019441

19. Loh CS, Sheng Y, Ifenthaler D. Serious games analytics: Theoretical Framework. In: Loh C, Sheng Y, Ifenthaler D, eds. Serious Games Analytics, Advances in Game-Based Learning. Springer Cham; 2015:3-29. doi:10.1007/9783-319-05834-4_1

20. Tan JW, Zary N. Diagnostic markers of user experience, play, and learning for digital serious games: a conceptual framework study. JMIR Serious Games. 2019;7(3):e14620. doi:10.2196/14620

21. Lu AS, Kharrazi H. A state-of-the-art systematic content analysis of games for health. Games Health J. 2018;7(1):1-15. doi:10.1089/g4h.2017.0095

22. Raiff BR, Jarvis BP, Rapoza D. Prevalence of video game use, cigarette smoking, and acceptability of a video gamebased smoking cessation intervention among online adults. Nicotine Tob Res. 1012;14(12):1453-1457. doi:10.1093/ntr/nts079

23. Derksen ME, van Strijp S, Kunst AE, Daams JG, Jaspers MWM, Fransen MP. Serious games for smoking prevention and cessation: A systematic review of game elements and game effects. J Am Med Inform Assoc. 2020:27(5):818-833. doi:10.1093/jamia/ocaa013

24. Krishnan N, Elf JL, Chon S, Golub JE. COach2Quit: A Pilot Randomized Controlled Trial of a Personal Carbon Monoxide Monitor for Smoking Cessation. Nicotine Tob Res. 2019;21(11):1573-1577. doi:10.1093/ntr/nty182

25. Marler JD, Fujii CA, Galanko JA, Balbierz DJ, Utley DS. Durability of Abstinence After Completing a Comprehensive Digital Smoking Cessation Program Incorporating a Mobile App, Breath Sensor, and Coaching: Cohort Study. J Med Internet Res. 2021;23(2):e25578. doi:10.2196/25578

26. Tan NC, Mohtar ZBM, Koh YLE, et al. An exhaled carbon monoxide self-monitoring device linked to social media to support smoking cessation: A proof of concept pilot study. Proceedings of Singapore Healthcare. 2018;27(3):187192. doi:10.1177/201010581857257

27. Raiff BR, Fortugno N, Scherlis NR, Rapoza D. A Mobile Game to Support Smoking Cessation: Prototype Assessment. JMIR Serious Games. 2018;6(2):e11. doi:10.2196/games.9599

28. Julious SA. Sample size of 12 per group rule of thumb for a pilot study. Pharmaceutical Statistics. 2005;4(4)287291. doi:10.1002/pst.185

29. Cugelman B. Gamification: what it is and why it matters to digital health behavior change developers. JMIR Serious Games. 2013;1(1):e3. doi:10.2196/games.3139

30. Heatherton TF, Kozlowski LT, Frecker RC, Fagerström KO. The Fagerström test for nicotine dependence: A revision of the Fagerström Tolerance Questionnaire. Br J Addict. 1991;86(9):1119-1127. doi:10.1111/j.1360-0443.1991.tb01879.x

31. Lairson DR, Harrist R, Martin DW, et al. Screening for patients with alcohol problems: severity of patients identified by CAGE. J Drug Educ. 1992;22(4):337-352. doi:10.2190/H8QV-KAYU-QBYH-1LN3

32. American Psychiatric Association. Diagnostic and statistical manual of mental disorders. 4th ed. American Psychiatric Association; 1994:181.

33. Miller NS. Nicotine addiction as a disease. In: Cocores JA, ed. The clinical management of nicotine dependence. Spring-Verlag; 1991:66-68.

34. Ijsselsteijn WA, de Kort YAW, Poels K. The Games Experience Questionnaire. Technische Universiteit Eindhoven; 2013. Accessed May 11, 2021. https:// research.tue.nl/en/publications/the-game-experiencequestionnaire

35. Brooke J. SUS: a ‘quick and dirty' usability scale. In: Jordan PW, Thomas B, Weerdmeester A, McClelland AL. Usability Evaluation in Industry. Taylor and Francis; 1995.

\section{ACKNOWLEDGEMENTS}

The authors thank the National Medical Research Council of Singapore for funding the development of the innovation and implementation of the study. They are also grateful to the management staff, Caris Tan and Patricia Kin, at the Research Department in SingHealth Polyclinics, for their assistance in seeking IRB approval, and providing logistic support for the procurement of equipment and administration of the funding.

\section{CONFLICTS OF INTEREST}

The authors have each completed and submitted an ICMJE form for disclosure of potential conflicts of interest. The authors declare that they have no competing interests, financial or otherwise, related to the current work. N. C. Tan reports funding by the Clinician Innovator Development Award by the National Medical Research Council of Singapore (grant number $\mathrm{MOH}-\mathrm{Cl}$ 18Nov-0002), related to the current work, and support from SingHealth Polyclinics for participation in the International Shared Decision Making Conference in Quebec, Canada, in July 2019. 


\section{FUNDING}

This work is supported by the Clinician Innovator Development Award given by the National Medical Research Council of Singapore (Grant number $\mathrm{MOH}-\mathrm{ClA} 18$ Nov-0002). The funding body will not be involved in the study design, data collection and analysis, results interpretation and manuscript writing in this trial.

\section{ETHICAL APPROVAL AND INFORMED CONSENT}

The SingHealth Institution Review Board approved the study (CIRB\#: 2020/2762). The trial is registered with the clinical trials registry in the United States National Library of Medicine (NCT04630340).

DATA AVAILABILITY

Data sharing is not applicable to this article as no new data were created.

\section{AUTHORS' CONTRIBUTIONS}

NCT conceptualized the innovation and drafted the manuscript. YLEK provided the biostatistics analytical plan. CCG, SN and AMT developed the e-Coaching curriculum. SBL, HCDT and SHL are the technical team members to develop the STEADES hardware and software. All authors reviewed and approved the manuscript.

\section{PROVENANCE AND PEER REVIEW}

Not commissioned; externally peer reviewed. 